\title{
A Novel Parameter Decomposition Approach for Recovering Poses of Distal Locking Holes from Single Calibrated Fluoroscopic Image ${ }^{\star}$
}

\author{
Guoyan Zheng and Xuan Zhang \\ MEM Research Center-ISTB, University of Bern, CH-3014, Bern, Switzerland \\ guoyan.zheng@ieee.org
}

\begin{abstract}
One of the most difficult steps of intramedullary nailing of femoral shaft fractures is distal locking - the insertion of distal transverse interlocking screws, for which it is necessary to know the position and orientation of the distal locking holes of the intramedullary nail. This paper presents a novel parameter decomposition approach for solving this problem using single calibrated X-ray image. The problem is formulated as a model-based optimal fitting process, where the to-be-optimized parameters are decomposed into two sets: (a) the angle between the nail axis and its projection on the imaging plane, and (b) the translation and rotation of the geometrical models of the distal locking holes around the nail axis. By using a hybrid optimization technique coupling an evolutionary strategy and a local search algorithm to find the optimal values of the latter set of parameters for any given value of the former one, we reduce the multiple-dimensional model-based optimal fitting problem to a one-dimensional search along a finite interval. We report the in-vitro experimental results, which demonstrate that the accuracy of our approach is adequate for successful distal locking of intramedullary nails.
\end{abstract}

Keywords: distal locking, fluoroscopy, pose estimation, parameter decomposition, hybrid optimization, model-based method.

\section{Introduction}

It has been recognized that one of the most difficult steps of intramedullary nailing of femoral shaft fractures is distal locking - the insertion of distal interlocking screws, for which it is necessary to know the positions and orientations of the distal locking holes of the intramedullary nail 1. Complicating the process of locating and inserting the distal interlocking screw is the nail deformation with insertion. It has been reported that deformation occurs in several planes due to medial-lateral and anterior-posterior flexion of the distal nail after it has been inserted. Deformation analysis of solid $9 \mathrm{~mm}$ femoral nails using a magnetic tracking system in a cadaveric study has shown lateral translations of $18.1 \pm$

\footnotetext{
* This work was supported in part by Swiss National Science Foundation through project NCCR CO-ME.
} 
$10.0 \mathrm{~mm}$, dorsal translations of $-3.1 \pm 4.3 \mathrm{~mm}$, and rotational deformation of -0.1 \pm 0.2 degrees for the center of the distal transverse locking holes [1]. The reason for the wide variations of the insertion-related femoral nail deformation is due to the fact that the nail has to deform to the shape of the medullary canal upon insertion. The shape of the canal varies widely from person to person. Therefore, it is very difficult, to determine what the resultant locations and orientations of the distal locking holes will be relative to their initial position before it is deformed. The surgeon depends heavily on intra-operative X-ray means in a conventional surgical procedure for providing precise locations and orientations of the distal locking holes. It requires positioning the axis of the fluoroscope perpendicular to the locking holes so that these holes appear perfectly circular in the images. This is achieved through a trial-and-error method and requires long time X-ray exposure for both the surgeon and patient. It has been reported that the surgeon's direct exposure to radiation for each conventional surgical procedure was 3 - 30 min, of which $31 \%$ - 51\% was used for distal locking [2].

The desire to target accurately with as little as possible X-ray exposure has led to various attempts to develop image-based methods for recovering the positions and orientations of the distal locking holes [3] 4] 5]. These methods require either multiple calibrated images or single image but with perfectly circular holes in the image, which normally requires the X-ray technician to use a try-and-move method several times to achieve.

This paper presents a novel parameter decomposition approach for solving this problem using single calibrated fluoroscopic image. We do not ask for an image with perfectly circular holes but we do put a constraint on its acquisition, i.e., the reduced patient shaft should be roughly parallel to the image intensifier of the fluoroscopy machine, which is much easier to be achieved intraoperatively. We then formulate the pose recovery as a model-based fitting problem and decompose the to-be-optimized parameters into two sets: (a) the angle between the nail axis and its projection on the imaging plane, and (b) the translation and rotation of the geometrical models of the locking holes around the nail axis. By using a hybrid optimization technique coupling an evolutionary strategy and a local search algorithm to find the optimal values of the latter set of parameters for each give value of the former one, we reduce the multiple-dimensional optimal fitting problem to a one-dimensional search along a finite interval.

The paper is organized as follows. Section 2 describes image calibration, geometrical models, and preprocessing. In Section 3, we describe the proposed approach in details. Section 4 presents our in-vitro experimental results, followed by conclusions in Section 5 .

\section{Image Calibration, Geometrical Models, and Preprocessing}

(1) Image Calibration: In reality, the proximal fragment, the distal fragment, and the nail may be treated as three rigid bodies and registered independently. The rigid transformations between these three rigid bodies can be trivially 
obtained from a navigator such as an optoelectronic tracker, a magnetic tracker, or even a medical robot. As this is not our focus in this paper, here we assume that the fractured femur has already been reduced and the proximal fragment and distal fragment are kept fixed relative to each other at the time of image acquisition. We also assume that the nail has been inserted till the distal end of the femur and has been locked proximally by screw so that the complete femur and the nail can be treated as one rigid body. A local coordinate system is established on this rigid body through a so-called dynamic reference base technique 6]. In the following description, let's denote this patient coordinate system as $A-C O S$. All computations are done in this reference coordinate sytem.

To relate a pixel in the two-dimensional (2D) projection image to $A-C O S$, the acquired image has to be calibrated for physical projection properties and be corrected for various types of distortion. We have chosen a weak-perspective pinhole camera model for modeling the $\mathrm{C}$-arm projection [7. Using such a camera model, a $2 \mathrm{D}$ pixel $V_{\mathrm{I}}$ is related to a three-dimensional (3D) point $V_{\mathrm{A}}$ by following equations:

$$
S_{\mathrm{A}}=\frac{\left(V_{\mathrm{A}}-f_{\mathrm{A}}\right)}{\left\|V_{\mathrm{A}}-f_{\mathrm{A}}\right\|} ; \text { and }\left[\begin{array}{c}
V_{\mathrm{I}, x} \\
V_{\mathrm{I}, y} \\
1
\end{array}\right]=\left[\begin{array}{cccc}
c_{\mathrm{A}, x} & c_{\mathrm{A}, y} & c_{\mathrm{A}, z} & p_{\mathrm{I}, x} \\
\mathrm{r}_{\mathrm{A}, x} & \mathrm{r}_{\mathrm{A}, y} & \mathrm{r}_{\mathrm{A}, z} & p_{\mathrm{I}, y} \\
0 & 0 & 0 & 1
\end{array}\right]\left[\begin{array}{c}
S_{\mathrm{A}, x} \\
S_{\mathrm{A}, y} \\
S_{\mathrm{A}, z} \\
1
\end{array}\right]
$$

where $\|\cdot\|$ means to calculate the length of a vector and the vectors $f_{\mathrm{A}}, r_{\mathrm{A}}, c_{\mathrm{A}}$, $p_{\text {I }}$ represent the position of focal point, the vector along image row increasing direction, the vector along image column increasing direction, and the $2 \mathrm{D}$ position of piercing point, respectively. They are projection parameters used to describe the projection properties of the $\mathrm{C}$-arm and can be calibrated preoperatively or intraoperatively.

Eq. (1) can be used for both forward and backward projections. For example, if we want to calculate the direction $S_{\mathrm{A}}$ of the forward projection ray of an image point $V_{\mathrm{I}}$, an additional constraint $\left\|S_{\mathrm{A}}\right\|=1$ can be used together with Eq. (1) to solve for it. The projection ray of point $V_{\mathrm{I}}$ is defined by the focal point and the direction $S_{\mathrm{A}}$.

The position of the imaging plane in $A-C O S$ and the focal length in our camera model is implicitly determined using the calibrated focal point $f_{\mathrm{A}}$ and the vectors $r_{\mathrm{A}}$ and $c_{\mathrm{A}}$. Any $2 \mathrm{D}$ image point $V_{\mathrm{I}}$ corresponds to a $3 \mathrm{D}$ spatial point $V_{\mathrm{A}}$ in this imaging plane, which is the intersection between its forward projection ray and this plane.

(2) Geometrical models: The distal part of an intramedullary nail containing the two distal locking holes, which is what we are interested in, is modeled as a cylinder (Fig. 1, left). The distance $L$ between the centers of the two distal locking holes can be accurately extracted from its product information. The geometrical model of each locking hole is represented by two circles as shown by Fig. 1, right, and is used later to simulate X-ray projections.

To obtain the coordinates of those points (visualized as red dots in Fig. 1, right) used to describe the model of the lcoking hole, a local coordinate system 


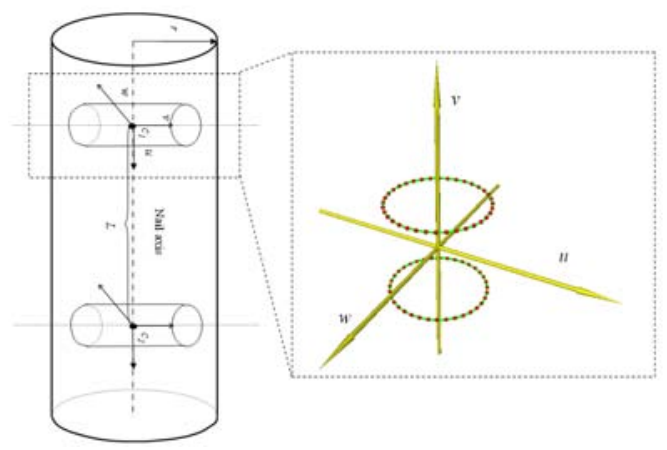

Fig. 1. The geometrical model of the distal part of the intramedullary nail (left) and the geometrical model of the distal locking hole (right)

$C^{\prime} u v w$ is established by taking the intersection point $C$ (it is also called the center of the locking hole) between the axis of the hole and the axis of the nail as the origin, the axis of the nail as the $u$ axis, and the axis of the locking hole as the $v$ axis (see Fig. 1 for details). The coordinates of those points expressed in this local coordinate system can be directly measured from the nail using a caliper, thanks to the symmetrical property of the locking hole; or extracted from the engineering drawings of the nail, if they are available.

(3) Preprocessing: The task of the preprocessing is to determine the projections of the distal locking holes. To extract these feature points from the image, Hough transform $[8$ is used to find the two mostly parallel edge lines of the projection of the distal part of the nail after applying a Canny edge detector to the image. The projection of the axis of the nail is considered as the middle line between these two mostly parallel edge lines. To determine those edge pixels belonging to the locking holes, the method reported in [5] is modified for our purpose. A parallelpiped window, whose sizes are equal to the distance between the detected edge lines, is swept along the middle line to find two locations which contain the maximum number of edge pixels and whose distance is greater than a pre-selected distance threshold $\tau$ (e.g. the width of the window). The centroids of the detected edge pixels in both locations are then calculated. The projection point of the center of each locking hole is then determined by finding the closest point on the middle line to the associated centroid. A preprocessing example is shown in Fig. 2.

\section{The Proposed Approach}

\subsection{Model-Based Fitting for Pose Recovery}

Using above detected feature points, we can find their corresponding spatial points on the imaging plane. Let's denote them as $d_{1}$ corresponding to the projection point of the center $C_{1}$ of the distal hole (the hole that is closer to the 


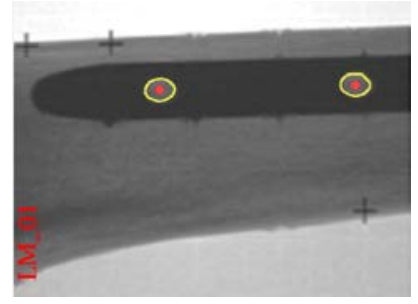

Fig. 2. A preprocessing example. The detected projection centers are displayed together with the extracted edge pixels of the distal locking holes.

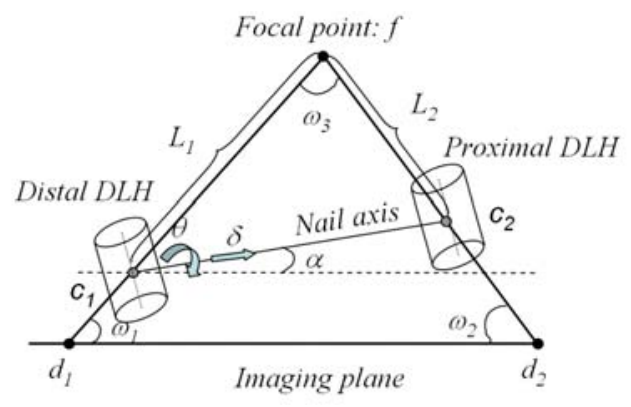

Fig. 3. Schematic view of the model-based fitting for pose recovery

nail tip), and $d_{2}$ corresponding to the projection point of the center $C_{2}$ of the proximal hole, respectively, as shown in Fig. 3. These two points define a line in $A-C O S$. This line together with the focal point $f$ defines a plane where the axis of the distal part of the nail should fall in. As we know the coordinates for point $f, d_{1}$, and $d_{2}$, we can calculate three internal angles $\omega_{1}, \omega_{2}, \omega_{3}$ of the triangle $f d_{1} d_{2}$. Assume the angle between the nail axis and its projection in the imaging plane is $\alpha$, then the coordinates of the centers of both locking holes are calculated as following:

$$
\begin{aligned}
& C_{1}=f+L_{1} \cdot \frac{\left(d_{1}-f\right)}{\left\|d_{1}-f\right\|} ; C_{2}=f+L_{2} \cdot \frac{\left(d_{2}-f\right)}{\left\|d_{2}-f\right\|} \\
& L_{1}=L \cdot \frac{\sin \left(\omega_{2}+\alpha\right)}{\sin \left(\omega_{3}\right)} ; L_{2}=L \cdot \frac{\sin \left(\omega_{2}+\alpha\right) \cdot \cos \left(\omega_{3}\right)}{\sin \left(\omega_{3}\right)}+L \cdot \cos \left(\omega_{2}+\alpha\right) \\
& \text { where } \alpha \in(-\pi / 2, \pi / 2)
\end{aligned}
$$

where $L$ is the distance between the centers of two holes. It can be measured or extracted from the product information.

Accoding to Eq. (2), the coordinates of both centers only depends on the parameter $\alpha$, so as the direction of the nail axis $\left[n_{x}, n_{y}, n_{z}\right]^{T}$.

Assuming that the coordinates of the center $C$ of one of the locking holes is denoted as $\left[C_{x}, C_{y}, C_{z}\right]^{T}$, the problem to estimate the pose of the locking hole in $A-C O S$ is now changed to find the rotation angle $\alpha$ of the nail axis, the rotation angle $\theta$ and the translation distance $\delta$ of the geometrical model of the distal locking hole along the nail axis so that the simulated projection can 
be fitted to its real X-ray projection (see Fig. 3 for details). This constrained transformation around the parameterized nail axis could be described by a $3 \times 3$ rotation matrix $\operatorname{rot}(\alpha, \theta, \delta)$ :

$$
\begin{aligned}
& {\left[\begin{array}{ccc}
n_{x}^{2}+S S_{y z} \cos (\theta) & M_{x y} \cdot C C-n_{z} \sin (\theta) & M_{z x} \cdot C C+n_{y} \sin (\theta) \\
M_{x y} \cdot C C+n_{z} \sin (\theta) & n_{y}^{2}+S S_{z x} \cos (\theta) & M_{y z} \cdot C C-n_{x} \sin (\theta) \\
M_{z x} \cdot C C-n_{y} \sin (\theta) & M_{y z} \cdot C C+n_{x} \sin (\theta) & n_{z}^{2}+S S_{x y} \cos (\theta)
\end{array}\right]} \\
& \text { where } S S_{x y}=n_{x}^{2}+n_{y}^{2} ; S S_{y z}=n_{y}^{2}+n_{z}^{2} ; S S_{z x}=n_{z}^{2}+n_{x}^{2} ; \\
& \text { and } M_{x y}=n_{x} n_{y} ; M_{y z}=n_{y} n_{z} ; M_{z x}=n_{z} n_{x} ; C C=1-\cos (\theta)
\end{aligned}
$$

and a translational vector $\operatorname{trans}(\alpha, \theta, \delta)=\left[t_{x}, t_{y}, t_{z}\right]^{T}$ :

$$
\left\{\begin{aligned}
& t_{x}=\left(C_{x}+\delta \cdot n_{x}\right) \cdot\left(n_{y}^{2}+n_{z}^{2}\right)-n_{x} \cdot\left(\left(C_{y}+\delta \cdot n_{y}\right) \cdot n_{y}+\left(C_{z}+\delta \cdot n_{z}\right) \cdot n_{z}\right)+ \\
&\left(n_{x} \cdot\left(\left(C_{y}+\delta \cdot n_{y}\right) \cdot n_{y}+\left(C_{z}+\delta \cdot n_{z}\right) \cdot n_{z}\right)-\left(C_{x}+\delta \cdot n_{x}\right)\right. \\
&\left.\cdot\left(n_{y}^{2}+n_{z}^{2}\right)\right) \cos (\theta)+\left(\left(C_{y}+\delta \cdot n_{y}\right) \cdot n_{z}-\left(C_{z}+\delta \cdot n_{z}\right) \cdot n_{y}\right) \sin (\theta) \\
& t_{y}=\left(C_{y}+\delta \cdot n_{y}\right) \cdot\left(n_{x}^{2}+n_{z}^{2}\right)-n_{y} \cdot\left(\left(C_{x}+\delta \cdot n_{x}\right) \cdot n_{x}+\left(C_{z}+\delta \cdot n_{z}\right) \cdot n_{z}\right)+ \\
&\left(n_{y} \cdot\left(\left(C_{x}+\delta \cdot n_{x}\right) \cdot n_{x}+\left(C_{z}+\delta \cdot n_{z}\right) \cdot n_{z}\right)-\left(C_{y}+\delta \cdot n_{y}\right)\right. \\
&\left.\cdot\left(n_{x}^{2}+n_{z}^{2}\right)\right) \cos (\theta)+\left(\left(C_{z}+\delta \cdot n_{z}\right) \cdot n_{x}-\left(C_{x}+\delta \cdot n_{x}\right) \cdot n_{z}\right) \sin (\theta) \\
& t_{z}=\left(C_{z}+\delta \cdot n_{z}\right) \cdot\left(n_{x}^{2}+n_{y}^{2}\right)-n_{z} \cdot\left(\left(C_{x}+\delta \cdot n_{x}\right) \cdot n_{x}+\left(C_{y}+\delta \cdot n_{y}\right) \cdot n_{y}\right)+ \\
&\left(n_{z} \cdot\left(\left(C_{x}+\delta \cdot n_{x}\right) \cdot n_{x}+\left(C_{y}+\delta \cdot n_{y}\right) \cdot n_{y}\right)-\left(C_{z}+\delta \cdot n_{z}\right)\right. \\
&\left.\left(n_{x}^{2}+n_{y}^{2}\right)\right) \cos (\theta)+\left(\left(C_{x}+\delta \cdot n_{x}\right) \cdot n_{y}-\left(C_{y z}+\delta \cdot n_{y}\right) \cdot n_{x}\right) \sin (\theta)
\end{aligned}\right.
$$

The pose recovery problem can then be formulated as an optimal model-based fitting:

$$
\underset{\left\{\alpha^{*}, \theta^{*}, \delta^{*}\right\}}{\arg \min } \sum_{i}\left\|e_{j=C P(i)}-\operatorname{Pr}\left(\operatorname{rot}(\alpha, \theta, \delta) \cdot m_{i}+\operatorname{trans}(\alpha, \theta, \delta)\right)\right\|^{2}
$$

where $\left\{e_{i}\right\}$ are the detected edge pixels of the locking holes; $\left\{m_{i}\right\}$ are the points used to describe their geometrical models; $\operatorname{Pr}(\cdot)$ denotes the projection operator; $C P(\cdot)$ denotes the action of finding the closest edge pixel of the simulated projection point into the image of a model point.

\subsection{Parameter Estimation}

Various techniques have been proposed for estimating parameters for modelbased fitting. Lowe [9] suggests to minimize the non-linear error function on image domain, where the perpendicular distance between projected model line and extracted edge point will be minimized. The correspondence between the model projection to image edge is found by selecting the one who has the shortest perpendicular distance. This strategy can lead to some ambiguity in fitting process when part of the model line has been occluded by structure of the model itself. This problem was solved by Fua [10] through applying hidden algorithm to avoid this pitfall. All these algorithms suffer from the facts that they are easily to be trapped by a local minimum and that the interpretation and initialization of model parameter values have to be done by the operator, which is not desirable for an intra-operative application in a sterilized environment. 
Parameter decomposition approach is a powerful optimization method that tries to decompose a high-dimensional problem into small, low-dimensional components and estimate the parameters for each component separately, thus reducing the computational complexity. The general idea of model decomposition for parameter estimation has bee successfully applied in many domains, e.g., geometrical curve fitting [11] and Bayesian model learning [12.

According to our observation that the size of the geometrical models of the distal locking holes (around $10 \mathrm{~mm}$ in each dimension) is relatively small compared to the focal length of the X-ray image (around $1000 \mathrm{~mm}$ ), we decompose the control parameters in Eq. (5) into two sets: (a) the angle $\alpha$ between the nail axis and its projection in the imaging plane; and (b) the rotation and translation distance of the geometrical models of the locking holes along the nail axis $(\theta, \delta)$. Now the original optimization problem can be re-formulated as:

$$
\underset{\alpha^{*}}{\arg \min }\left[\left(\underset{\left\{\theta^{*}, \delta^{*}\right\}}{\arg \min } \sum_{i}\left\|e_{j=C P(i)}-\operatorname{Pr}\left(\operatorname{rot}(\alpha, \theta, \delta) \cdot m_{i}+\operatorname{trans}(\alpha, \theta, \delta)\right)\right\|^{2}\right)\right]
$$

Where the term in the square brackets simply means the minimum sum of distance for a fixed $\alpha$ and all possibilities of $(\theta, \delta)$. The advantage of such decomposition lies in the fact that the latter set of parameters can be calculated by using a hybrid optimization technique coupling an evolutionary strategy and an iterative closest projection point algorithm (ICPP) as described below, which then reduces the original multiple-dimensional optimization problem to a onedimensional search in a finite interval.

A. Initialization: Given a fixed $\alpha$, we can estimate the positions of both centers of the locking holes and the orientation of the nail axis. Then, the initial transformation between the local coordinate system of the geometrical model of the locking holes and $A-C O S$ can be obtained by taking the estimated center as the origin, the estimated nail axis as the $u$ axis, and the normal of the imaging plane as the $v$ axis. All points defined in the local coordinate system of the geometrical model can then be transformed to $A-C O S$ using this transformation. The optimal values of the rotation $\theta$ and the translation $\delta$ of the models along the nail axis can be optimally estimated by fitting the geometrical models of the locking holes to the image as by a hybrid optimization technique as described below.

B. The Iterative Closest Projection Point (ICPP) Algorithm: Let us denote $E$ be a set of $N_{E}$ detected $2 \mathrm{D}$ edge pixels $\left\{e_{1}, e_{2}, \ldots, e_{N_{E}}\right\}$ of the locking hole projection. Further denote $M^{t-1}$ be a set of $N_{M}$ model point $\left\{m_{0}^{t-1}, m_{1}^{t-1}, \ldots, m_{N_{M}}^{t-1}\right\}$ at iteration step $t-1$. Now in the iteration step $t$, we perform following steps:

Simulating $X$-ray projection: In this step, we simulate the X-ray projection of the geometrical models of the locking holes to remove invisible points. Let $P^{t-1}$ be a set of $N_{P} 2 \mathrm{D}$ projection points $\left\{p_{1}^{t-1}, p_{2}^{t-1}, \cdots, p_{N_{P}}^{t-1}\right\}$ obtained by simulating X-ray projection of the $3 \mathrm{D}$ models into the image. Normally $N_{P}<<N_{M}$. Thus, for each $2 \mathrm{D}$ projection point $p_{i}^{t-1}$, we know its associated $3 \mathrm{D}$ model point $m_{i}^{t-1}$. 
Find closest projection point: In this step, we try to find the closest neighbor edge pixel $e_{i}$ of each 2D model projection point $p_{i}^{t-1}$.

Establishing 3D-2D correspondence: For each 2D matched pairs $\left(e_{i}, p_{i}^{t-1}\right)$, calculate the forward projection ray $B P_{i}$ of the $2 \mathrm{D}$ edge pixel $e_{i}$. Then for the ray $B P_{i}$, calculate a $3 \mathrm{D}$ point pair $P P_{i}^{t-1}=\left(b e_{i}^{t-1}, m_{i}^{t-1}\right)$, where $b e_{i}^{t-1}$ is a point on the line $B P_{i}$ that is closest to the $3 \mathrm{D}$ model point $m_{i}^{t-1}$ of the model projection point $p_{i}^{t-1}$.

Estimating pose: For all calculated 3D point pairs $P P S^{(t-1)}=\left\{P P_{i}^{t-1}\right\}$, find an optimal local solution of all pose parameters by minimizing following cost function:

$$
\begin{gathered}
\underset{\left\{\theta^{(t-1) *}, \delta^{(t-1) *}\right\}}{\arg \min } S\left(\theta^{(t-1)}, \delta^{(t-1)}\right) ; \text { where } S\left(\theta^{(t-1)}, \delta^{(t-1)}\right)= \\
\sum_{i} \| b e_{i}^{t-1}-\left(\operatorname{rot}\left(\theta^{(t-1)}, \delta^{(t-1)}\right) \cdot m_{i}^{t-1}+\operatorname{trans}\left(\theta^{(t-1)}, \delta^{(t-1)}\right) \|^{2}\right.
\end{gathered}
$$

where we drop the symbol $\alpha$ from the expressions, as its value is fixed.

These steps are repeated until all pose parameters are converged.

C. The Evolutionary Strategy: The ICPP algorithm can be regarded as a local minimum search algorithm but we are trying to find the global minimum of the disparity function that may be well hidden among many poorer local minima, especially when a poor initialization is used. In our approach, this is handled by combining a conventional genetic algorithm 13 with the ICPP algorithm. The genetic algorithm acts as a random generator for possible parameter sets that solve the minimization problem. All generated individual parameter set is then fed through the ICPP algorithm before being rated using the disparity function. Five best ones (with the lowest values of $S\left(\theta^{(t-1)}, \delta^{(t-1)}\right)$ ) become the parents of next generation. The algorithm stops when the differences of the disparity function values of all five best ones are smaller than a pre-selected threshold.

D. Optimization of parameter $\alpha$ : We now convert a multiple-dimensional optimization problem to a one-dimensional one, where the parameter $\alpha$ can be optimized by a search along a finite interval $\left[-30^{\circ},+30^{\circ}\right]$ (due to the acquisition constraint that the nail should be put roughly parallel to the imaging plane). A typical optimization space of this parameter is shown in Fig. 4. It has a symmetrical shape and a clear global optimum around the ground truth $\alpha=10.4^{\circ}$. We could separate the optimization space into two sub-intervals, i.e. $\left[-30^{\circ}, 0\right)$ and $\left[0,30^{\circ}\right]$. In each sub-interval, the optimum of that sub-interval could be easily found by a local search algorithm starting from any initialization value. Actually, in all experiments, we have simply initialized $\alpha$ by the middle value of each sub-interval. The global minimum is then found by taking the better one of the two optima

\section{Experimental Results}

We design and conducted in vitro experiments to analyze the accuracy and robustness of the proposed approach. A SYNTHES৫) (STRATEC Medical, 


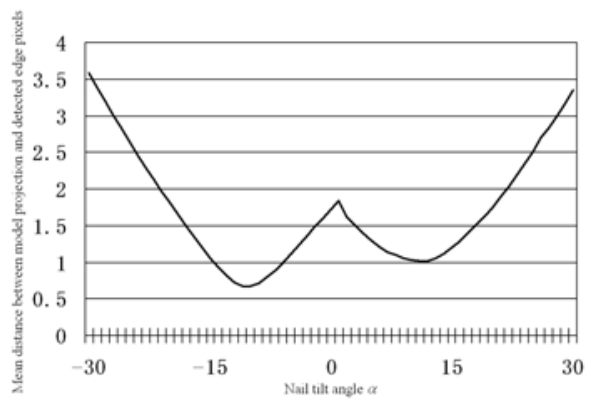

Fig. 4. Optimization space of the parameter $\alpha$

Oberdorf, Switzerland) $9 \mathrm{~mm}$ solid titanium femoral nail was used in our study. It was inserted into a cadaveric human femur and was locked proximally. A Siemens ISO-C C-arm (Siemens AG, Erlangen, Germany) was used to acquire fluoroscopic images for our experiments. The ground truth of the positions of the locking holes was obtained after image acquisition by inserting a custom-made steel rod through the hole and then digitizing both top and bottom centers of the rod using an optically trackable sharp pointer (OPTOTRAK 3020, Northern Digital Inc, Waterloo, Canada).

Three images acquired from different directions were used in our experiments, as shown in Fig. 5. For each image, we applied the proposed approach ten times to estimate the poses of the distal locking holes. The estimated results were compared to the ground truth to compute the errors for each hole, which were defined as the angular difference between the estimated hole axis and the one obtained through pointer-based digitization and the positional difference of the entry point and its ground truth along the plane perpendicular to the hole axis, because the positional difference along the hole axis is not important for the task for insertion of distal locking screws.

In all studies, the poses of the distal locking holes could be automatically recovered. The angular and positional errors are shown in Table 1. Compared to ground truths, the average angular error was found to be $1.0^{\circ}\left(\operatorname{std}=0.4^{\circ}\right)$ and the average positional error along the plane perpendicular to the hole axis was found to be $0.6 \mathrm{~mm}(\mathrm{std}=0.4 \mathrm{~mm})$.

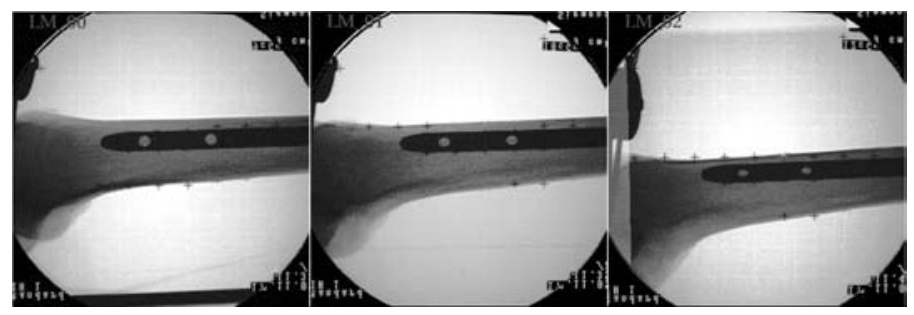

Fig. 5. Three images used in the in - vitro experiment: LM_00, LM_01, LM_02 
Table 1. In - vitro experimetal results

\begin{tabular}{l|c|c}
\hline Image & Angular differences $\left(^{\circ}\right)$ & Positional differences $(\mathrm{mm})$ \\
\hline LM_00 & $0.7 \pm 0.3$ & $0.2 \pm 0.0$ \\
\hline LM_01 & $0.9 \pm 0.2$ & $0.4 \pm 0.1$ \\
\hline LM_01 & $1.5 \pm 0.2$ & $1.1 \pm 0.1$ \\
\hline Overall & $1.0 \pm 0.4$ & $0.6 \pm 0.4$ \\
\hline
\end{tabular}

\section{Conclusions}

We have presented a novel parameter decomposition approach for automatic pose recovery of distal locking holes from single calibrated fluoroscopic image. Unlike previously introduced method [5], our approach does not ask for an image with perfectly circular holes. Our in-vitro experimental results demonstrate that the accuracy of our approach is adequate for successful distal locking of intramedullary nails.

\section{References}

1. Krettek, C., Mann, J., Miclau, T. et al.: Deformation of femoral nails with intramedullary insertion. J. Orthop. Res. 16, 572-675 (1998)

2. Skjeldal, S., Backe, S.: Interlocking medullary nails - radiation doses in distal targeting. Arch Orthopaedic Trauma Surg. 106, 179-181 (1987)

3. Zhu, Y., Phillips, R., Griffiths, J.G., et al.: Recovery of distal hole axis in intramedullary nail trajectory planning. In: Proc Inst Mech Eng [H], vol. 216, pp. 323-332 (2002)

4. Leloup, T., Schuind, F., Warzee, N.: Process for the acquisition of information intended for the insertion of a locking screw into an orifice of an endomedullary device. European Patent Application Number: 04447153.0 (2004)

5. Yaniv, Z., Joskowicz, L.: Precise robot-assisted guide positioning for distal locking of intramedullary nails. IEEE Trans Med. Imaging 24, 624-635 (2005)

6. Nolte, L.-P., Visarius, H., Arm, E. et al.: Computer-aided fixation of spinal implants. J. Image Guid Surg. 1, 88-93 (1995)

7. Gremban, K.D., Thorpe, C.E., Kanade, T.: Geometric camera calibration using systems of linear equations. In: Proceedings of IEEE conference on robotics and automation, pp. 562-567(1988)

8. Jain, R., Kasturi, R., Schunk, B.G.: Machine Vision. McGraw-Hill, New York (1995)

9. Lowe, F.G.: Fitting parameterized three-dimensional models to images. IEEE Trans Pattern Anal Mach Intell 13, 441-450 (1991)

10. Fua, P.: Model-based optimization: accurate and consistent site modeling. In: Proceedings of the 18th Congress, International Society for Photogrammetry and remote sensing, pp. 222-223 (1996)

11. Jiang, X., Cheng, D.C.: A novel parameter decomposition approach to faithful fitting of quadric surfaces. In: Kropatsch, W.G., Sablatnig, R., Hanbury, A. (eds.) Pattern Recognition. LNCS, vol. 3663, pp. 168-175. Springer, Heidelberg (2005)

12. Neapolitan, R.E.: Learning Bayesian Networks (1st Ed). Prentice Hall

13. Goldberg, D.E.: Genetic algorithms in search, optimization, and machine learning, Reading, MA. Addison-Wesley, London (1989) 